

\title{
Social Pharmacology: Expanding horizons
}

\author{
Rituparna Maiti, José-Luis Alloza'
}

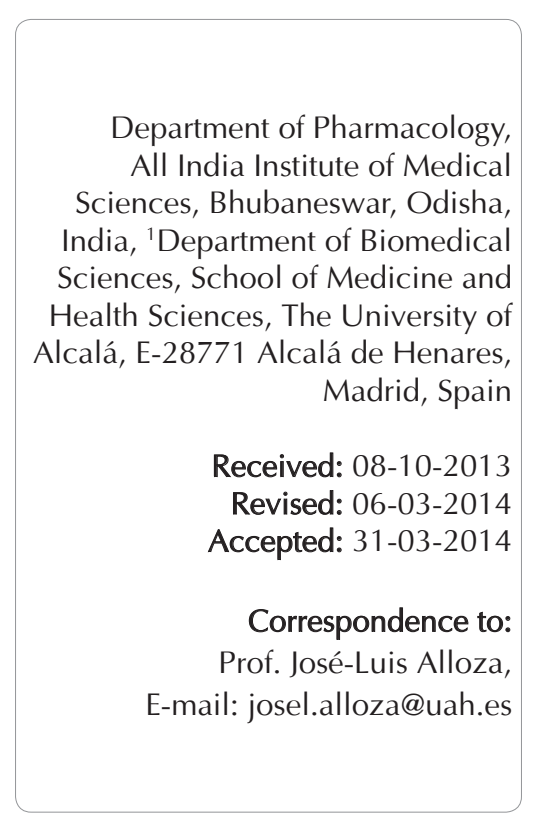

\begin{abstract}
In the current modern and global society, social changes are in constant evolution due to scientific progress (technology, culture, customs, and hygiene) and produce the freedom in individuals to take decisions by themselves or with their doctors toward drug consumption. In the arena of marketed drug products which includes society, individual, administration, and pharmaceutical industry, the young discipline emerged is social pharmacology or sociopharmacology. This science arises from clinical pharmacology, and deals with different parameters, which are important in creating knowledge on marketed drugs. However, the scope of "social pharmacology" is not covered by the so-called "Phase IV" alone, but it is the science that handles the postmarketing knowledge of drugs. The social pharmacology studies the "life cycle" of any marketed pharmaceutical product in the social terrain, and evaluates the effects of the real environment under circumstances totally different in the drug development process. Therefore, there are far-reaching horizons, plural, and shared predictions among health professionals and other, for beneficial use of a drug, toward maximizing the benefits of therapy, while minimizing negative social consequences.
\end{abstract}

KEY WORDS: Clinical pharmacology, drug abuse, Phase IV, post marketing period, sociopharmacology

\section{Introduction}

Social pharmacology or sociopharmacology, a relatively new field in clinical pharmacology, depicts the relationships between society and drugs. In its development, the name "sociopharmacology" was coined in 1960s when investigators realized that it was necessary to assess the effects of drug addiction on the mood and behavior of individuals in social settings (primarily psychotropic and drug abuse agents). ${ }^{|1-3|}$ This term currently is also used by several researchers restricted to drug abuse circumstances, and social behavior impact. . $^{4,5]}$ The new approach of social pharmacology and its development started in 1980s. During the past few decades, the concept of social pharmacology has evolved, and this new discipline explores the multiple dimensions of drug use during the postmarketing period. Thus, this discipline has expanded its horizon and enriched the specialty by incorporating the contributions from physicians, pharmacists, nurses, biologists,

\begin{tabular}{|l|c|}
\hline \multicolumn{2}{|c|}{ Access this article online } \\
\hline Website: www.ijp-online.com & Quick Response Code: \\
\hline DOl: 10.4103/0253-7613.132151 & \\
\hline &
\end{tabular}

drug epidemiologists, health economists, lawyers, regulators, insurance specialists, and communications specialists. ${ }^{[6-14]}$

Social pharmacology as a field of health science generates knowledge of marketed drugs in the modern world; it refers to the evaluation of the social consequences of an individual's exposure to any marketed drug and the factors related to drug utilization. This discipline accomplishes the goal of studying how to attain knowledge still to be discovered when a drug is marketed, because the approval dossier is a tiny part of the knowledge that remains to be gathered. The scenario of the postmarketing period represents the widest scope of vision of medications when they are studied in their current "life cycle" in the social habitat, because of their new specific and aggressive environment after approval when the strict control of the drug development process is no longer in place, or due factors related to owners of a drug molecular entity, the operations and management by the pharmaceutical industry, and the regulators. With regard to public health for the individual and society, social pharmacology is perceived as the tip of the iceberg of what we could explore to obtain essential data for any marketed drug, with the contribution of the heterogeneous expertise of health professions. The wide scope of interactions among different and extensive vectors which are linked to use and benefits of marketed drugs gives this subject huge potential in drawing conclusions about risk-benefit factors, providing alert responses, proposing actions for the decision making 
process, minimizing the negative impact of drugs, as well as promoting the proper and efficient use of drugs. Therefore, the scope of "social pharmacology" is not covered by the so-called "Phase IV" alone.

\section{The Concept}

The researchers in sociology and psychology conceptualized social pharmacology to describe the use of psychoactive drugs and drug dependence. However, today social pharmacology is a vast discipline which gives special emphasis on the postmarketing period of drugs and other medicinal products. ${ }^{[12]}$

Venulet states that social pharmacology is "the ultimate step in the natural history of pharmacology in which the properties of a drug, its availability, doctor's prescribing patterns, patient's compliance, etc., combine and interact in a manner which determines the final effects of the therapeutic efforts". ${ }^{[6,15]}$ The author (Alloza) defines "social pharmacology as a multidisciplinary science and dynamic system that harmonizes the critical and influential elements in the use of marketed drugs and the interrelationships, among health professionals, who directly or indirectly participate in health care, either by providing a service or improving drug therapy". ${ }^{12]}$

When focusing on a drug product there is a big difference between two situations: The postmarketing setting of a new drug is completely different from a drug development stage in which rigorous scientific methods are employed. There is a great disparity between ideal clinical research conditions during the clinical development of the drug and those of its new "social habitat" in which it will be exposed for use. External circumstances from the point of view of the consumer and society have to be taken into consideration because there may be differences in the cultural background such as health, hygiene, education, ability to understand, and inter-individual response, which creates a pool of inputs for broad horizons about drug use, including those social factors, which could explain how and why drugs are used outside clinical and rational healthcare frameworks. Thus, social pharmacology studies the marketed drug in a pluralistic society within a multidisciplinary structure. This means including all types of professionals who directly or indirectly participate in health care and public health, either by providing a service or improving drug therapy (information, communication, education, and problem solving). Therefore, the list of methodological approaches is very extensive (pharmacoepidemiological studies in drug surveillance, experimental and observational studies ["naturalistic"], drug response variation, outcome research, pharmacoeconomic studies, drug-toxicity evaluation, drug regulation evaluation, drug information evaluation, etc.).

\section{The Players}

Being an interdisciplinary science, social pharmacology is based on complex interrelationships. The major participants have been represented through an equilateral triangle, as shown in Figure 1. The drug product is represented as the center of interest and the three main players (health professionals, health authorities, and the pharmaceutical industry) are represented at the vertices. The relationship between patient/consumer and each of these three vertices occurs in different proportions.
Figure 1: The major players in sociopharmacology (Source: Alloza JL. Social Pharmacology: Conceptual remarks. Drug Information Journal 2004; 38: 321-329)

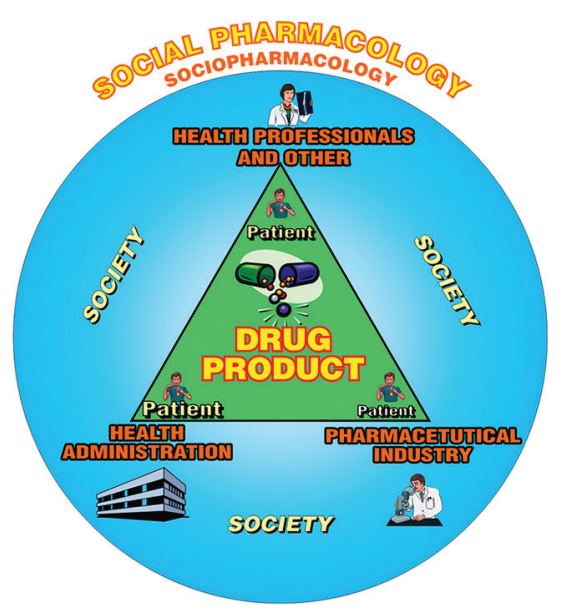

Many vectors can connect with different components and explores options for future research. ${ }^{[12]}$ The pharmaceutical industry should play a crucial role in the discovery of new drugs, which are medically and socially desirable. Pharmaceutical companies are also concerned in this domain for providing drug information to doctors as well as to patients. Regulatory agencies are involved in drug approval, regulation of the optimal level of drug use, application and translation of clinical trials to daily therapeutic practice. Health professionals scrutinize the rationale for prescription and self-medication of drugs. The role of the health professionals is to identify the social and irrational factors governing drug use in order to accustom and rationalize drug utilization in daily clinical practice. Drug utilization differs according to social characteristics of physicians (sub-specialty, medical education, cultural origin, etc.) or patients (gender, age, education, country, job, social status, etc.). ${ }^{[10]}$

\section{The Variables}

Social pharmacology refers to those variables that have a profound impact on drug action and the occurrence of specific drug effects as recognized and interpreted by the user. Previous studies have identified a number of variables (social and cultural factors) that influence the nature and occurrence of drug effects. Some important and well-studied variables include: Prior mood and body state, physical and social setting, symptom sensitivity, knowledge and information, body image and awareness, instructions/descriptions prior to use, suggestibility, judgments about drug effectiveness, personality, collective social knowledge about effects, ethnicity/ social-cultural background, social interaction dynamics, past experiences, social and communication networks, attitudes, accessibility and availability of drugs, expectations, health provider attitudes and perceptions, environmental cues, group behavior/tasks, modeling behaviors, motivations for use, rituals of preparation, administration, use, meanings of drug effects, value systems/self-image/lifestyle, labels for describing drug effects, external life events, interpretation of drug effects, social and legal controls over use, etc. Therefore in a global 
Figure 2: The mission of social pharmacology (Source: Alloza JL. Social Pharmacology: Conceptual remarks. Drug Information Journal 2004;38:321-329)

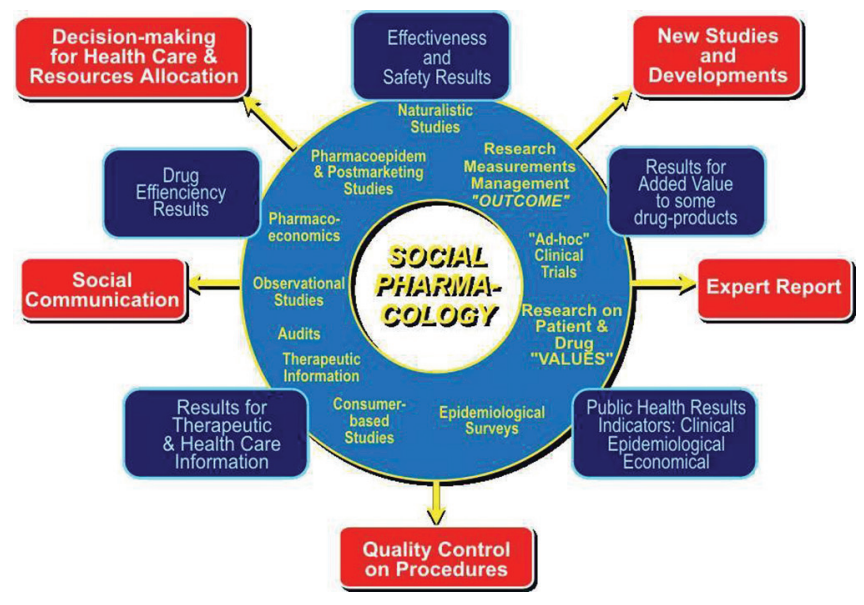

conception, the number characteristics for similar variables are widely distributed. ${ }^{[5]}$

\section{Ethnocultural background and Drug Use}

Culture is bigger than individual as it is related to mental health and substance use on several different strata. Community members from different social, ethnic or cultural groups may have a greater risk of mental illness or substance abuse problems because they may be more exposed to stressful conditions like discrimination and isolation. They may have divergent viewpoint of health and may face linguistic hurdles when they access health or other services.

Sociocultural faith and opinions can frame the approach to and behavior regarding drug use and abuse. Culture plays a pivotal role in shaping the mind set of individuals about potential problems they may face with substance use. ${ }^{[16]}$ For many sociocultural bodies, this may grant a protection. For example, the ancient Aztecs used to take alcohol before any contact with white colonists or immigrants. Their alcohol use was restricted and was only for rituals and otherwise use of alcohol was strictly punished under penalty of death. ${ }^{[17]}$ Another example is the development of the peyote sect in Northern Mexico. Peyote was used in a ceremonial backdrop to treat alcohol addiction. This use later became an essential part of the native American church, which offered important metaphysical treatment for chronic alcoholism. ${ }^{[17]}$ Beginning of excessive substance use may occur during periods of rapid social change, often among a race who had little access to a drug before. Loss of a healthy ethnocultural integrity may occur among indigenous populations whose cultures have been ruined by the pervasive and abrupt invasion of foreign influence. ${ }^{[18]}$

Use of psychoactive drugs and substance-abuse behavior can be attributed to genetic and cultural background. The rise in drug abuse and addiction is a real concern in India. Recent surveys reveal that India has approximately 70 million drug addicts. In India, the cultural consciences are changing, the underprivileged class is suffering with economic oppression while there is an enormous rise in the upper socioeconomic class, all of this combined with the declining support of the family which is leading to drug abuse and addiction. In India, heroin and alcohol are the most abused drugs. However, use of drugs like methamphetamine, cocaine, and crack are on the rise throughout India. India has witnessed a rise in industrialization and urbanization over the past two decades leading to large migrations to its cities. This is gradually fading traditional culture and changing lifestyle of individual Indians. Their new life is making them very susceptible to the stresses and strains of modern urban life and to deal with this situation a person is turning to drugs for shortterm pleasure. Since, there is a rise in drug abuse and addiction in India, the country has noticed an increase in transmitted diseases such as HIV, tuberculosis, hepatitis B and C, etc., which is a major concern of the healthcare sector. ${ }^{[19]}$

\section{Studies in Social Pharmacology}

Different kinds of research questions may arise with respect to interrelationships between (a) social and physical environmental variables, (b) biochemical and physiological states, and (c) pharmacological agents. The majority of studies done in sociopharmacology are focused on the effect of pharmacological agents on social behaviors. These studies provide organization for a diverse and complex field of investigation and suggest the kinds of relationships which require investigation. At the same time, it has some weaknesses also. Genetic contributions are not easily included because phenotypic characters result from interactions between genotype and the physical and social environments. Further, social behavior is poorly understood as it is known that in some circumstances, altering group composition alters drug responses in individuals. ${ }^{[3]}$

\section{The Mission}

Government and health authorities can utilize the results of social pharmacology studies in decision-making regarding effectiveness, safety, therapeutic efficacy, public health indicators, treatment information, and resource allocation. In addition, social pharmacology generates hypotheses for new studies and enhances quality control of existing procedures. ${ }^{[12]}$ Social pharmacology also contributes by making a framework for identifying, classifying and analyzing variables and understanding the mechanism of drug action in humans. It is the fundamental approach for integrating pharmaceutical and sociocultural variables to aid in clarifying and understanding user-generated portrayal of their drug experiences, with the objective of improving outcomes of drug therapy. The growing interest in social pharmacology has given an opportunity for pharmaceutical and social science researchers to explore the nature and meaning of drug-taking experiences in an integrated manner [Figure 2]. It is also important to teach this information to student and practicing pharmacists, other health professionals, social scientists and many other interested groups who work with drugs and consumers of drugs on a regular basis. ${ }^{[5]}$

\section{The Methods and Examples}

Social pharmacology utilizes the following established methods for evaluating the use of medicinal products and their consequences:

- Pharmacoepidemiologic studies: To assess the pattern of prescription, consumption, and consequences. ${ }^{[20]}$ 
Studies are also done to evaluate quality improvement of prescription, dispensing, and follow-up

- Experimental studies and observational studies: To obtain baseline data or direct evidence

- Naturalistic studies: For clinical evaluation by observing subjects in their natural environment ${ }^{[21]}$

- Longitudinal studies: To study the disease process and its development and longterm efficacy of the drugs

- Postmarketing surveillance studies: To evaluate the safety and efficacy of the already marketed drugs as well as overuse and misuse of drugs ${ }^{\mid 22]}$

- Drug utilization studies ${ }^{[23]}$

- Clinical audits and other overall assessment of healthcare ${ }^{[24]}$

- Studies on compliance of treatment ${ }^{[25]}$

- Development of treatment formularies and therapeutic guidelines: Comparative evaluation is done on the role of different criteria in developing the drug formulary therapeutic guidelines ${ }^{[26,27]}$

- Assessment of completeness and practical value of product package inserts

- Pharmacoeconomic studies: To describe and analyze the costs of drug therapy to health care systems and society ${ }^{283}$

- Research on outcomes, change in quality of life, and patient satisfaction $^{[29]}$

- Toxicity evaluation: Evaluation of acute drug toxicity, toxicity in special populations, and evaluation of the practical information available on the toxicity

- Analysis of drug interactions: With food, nutraceuticals, probiotics, and all other medicinal products

- Studies on the prevention of iatrogeny: Study on potential iatrogenic drugs and modes of prevention ${ }^{[30]}$

- Pharmacogenetic studies: To know and explain the variability in response due to ethnic variation ${ }^{[31]}$

- Chronopharmacological studies: To adjust the dosage or to decide proper dosing time depending on human biological rhythms ${ }^{132]}$

- Studies on special population: Use of drugs by at risk populations, especially pregnant women, nursing mothers, infants and children, the elderly, patients with chronic diseases (e.g., diabetes, renal, hepatic, cardiovascular, and psychiatric diseases) $)^{[33]}$

- Studies on medication error: To classify and analyze the errors in the use, prescription, dispensing, and formulation of drugs ${ }^{[34]}$

- $\quad$ Studies of drug compounding ${ }^{\mid 35]}$

- Epidemiological studies on self-medication, over-the-counter drugs and use of herbal medicines ${ }^{[36]}$

- Studies of the pattern of use and interactions among scientific medicine, quack-remedies, alternative medicine, and placebo effects ${ }^{[37]}$

- Evaluation of interrelationships between patients, healthcare professionals and the pharmaceutical industry ${ }^{[38]}$

- Evaluation of the quality of health information and patient health education on the appropriate use of medicinal products $^{[39]}$

- Evaluation of awareness on health care by the media (television, radio, newspaper) ${ }^{\mid 40]}$

- Systematic evaluation of accuracy of information available on the internet regarding medicinal products ${ }^{141]}$
- Assessment of impact on and attitude of the society toward new drugs

- The study of prescribing "off-label" use of medicines ${ }^{[42]}$

- Studies on counterfeit drugs

- Analysis of inter-professional communication among physicians, clinical pharmacologists, pharmacists, and patients $^{[43]}$

- Analysis of promotional drug literature from the pharmaceutical industry and its impact ${ }^{[44]}$

- Studies on natural products, banned substances, and doping agents

- Assessment of implementation and impact of rules and regulations made by different health authorities and regulatory bodies ${ }^{45,46]}$

- Analysis of the advantages and disadvantages of electronic medical material (prescription, medical records, protocols, and information) $)^{[47}$

- Studies on ethical guidelines for the protection of end users. $^{[48]}$

\section{Conclusion}

Today social pharmacology is a well-established discipline, included in the academic curriculum of medicine in some countries, with a remarkable role in the postmarketing period. Postmarketing period is a phase when it is possible to develop a large number of pharmacological, epidemiological and public health studies, aligned to assess the influence of the drugs over the individual and society, to provide vigilant feedbacks, as well as to recommend actions for the decision making process, in a substantial scientific basis which relates health professionals, health managers, administrators and the pharmaceutical industry. Thus, sociopharmacology brings together a vast assemblage of disciplines connected to evaluation of efficacy, safety, compliance, self-medication, and utilization of economic assets associated with the use of pharmaceutical products. To strengthen and enrich the discipline, future research should trace how social, economic and health policies create conditions that increase harmful drug use, as well as develop better ways to provide resources to individuals and communities to reduce harmful drug use.

\section{References}

1. Lennard HL. A proposed program of research in sociopharmacology. In: Leiderman PH, Shapiro D, editors. Psychobiological Approaches to Social Behaviour. California, USA: Stanford University Press; 1964. p. 127-37.

2. Barchas PR, Barchas JD. Sociopharmacology. In: Barchas JD, Barchas PR editors. Psychopharmacology: From Theory to Practice. NY: Oxford University Press; 1977. p. 80-7.

3. McGuire MT, Raleigh MJ, Brammer GL. Sociopharmacology. Annu Rev Pharmacol Toxicol 1982;22:643-61.

4. Morgan JP, Zimmer L. The social pharmacology of smokeable cocaine: Not all it's craked up to be. In: Reinarman C, Levine HG, editors. Crak in America: Demon Drugs and Social Justice. Ch. 7. California, USA: The Regents of the University of California; 1997.

5. Montagne M. Social pharmacology: Integrating pharmaceutical and social science research on drug effects. Drug Inf J 2004;38:315-20.

6. Venulet J. Aspects of social pharmacology. In: Jucker J, editor. Progress in Drug Research. Vol. 22. Basel, Stuttgart: Bikhäuser Verlag; 1978. p. 10-25.

7. Alloza JL. Family medicine, epidemiological drug research and social pharmacology. Med Clin (Barc) 1984;82:124-9.

8. Alloza JL. Epidemiological drug surveys in the ambulatory care environment. In: Alloza JL, editor. Clinical and Social Pharmacology. Postmarketing Period. Aulendorf, Germany: Editio Cantor; 1985. p. 38-56. 
9. Alloza JL. "Social Pharmacology, a New Discipline" (Tribune), Diario Médico (The Medical Journal, Madrid, Spain), May $10^{\text {th }}, 2000$ : page 8.

10. Montastruc JL. Social pharmacology: A new topic in clinical pharmacology. Therapie 2002;57:420-6.

11. Alloza JL. Social pharmacology, the tip of the iceberg in public health. Methods Find Exp Clin Pharmacol 2003;25 Suppl A: 175.

12. Alloza JL. Social pharmacology: Conceptual remarks. Drug Inf J 2004;38:321-9.

13. Alloza JL. Social pharmacology: The discipline that studies the marketed drug and its environment. Doctor (El Medico) 2006;970:9-14

14. Mbongue TB, SommetA, PathakA, Montastruc JL. "Medicamentation" of society, non-diseases and non-medications: A point of view from social pharmacology. Eur J Clin Pharmacol 2005:61:309-13.

15. Venulet J. Towards social pharmacology. In: Alloza JL, editor. Clinical and Social Pharmacology. Post Marketing Period. Aulendorf, Germany: Editio Cantor; 1985. p. 129-39.

16. Heath DB. Culture and substance abuse. Psychiatr Clin North Am 2001;24:479-96, vii-viii.

17. Abbott PJ. American Indian and Alaska native aboriginal use of alcohol in the United States. Am Indian Alsk Native Ment Health Res 1996;7:1-13.

18. Westermeyer J. Cross-cultural aspects of substance abuse. In: Galanter M, Kleber HD, editors. Textbook of Substance Abuse Treatment. Arlington, VA: American Psychiatric Publishing; 2004. p. 89-98.

19. Mordey A. Drug abuse and addiction in India. Available from: http://www. thecabinchiangmai.com/archive/drug_abuse_and_addiction_in_india. [Last accessed on 25th September 2013]

20. Experimental Technology Incentives Program. The Experiment in Post-Marketing Surveillance of Prescription Drugs: An Initial Status Report. Washington DC: National Bureau of Standards; 1982.

21. Alloza JL. A naturalistic study on post-operative acute pain: Comparison between two standard narcotic analgesics (morphine and meperidine). Pain (Dolor) 1987;2:55-61.

22. Temple RJ, Jones JK, Crout JR. Adverse effects of newly marketed drugs. N Engl J Med 1979;300:1046-7.

23. Rucker TD. Drug utilization review: Guidelines for program development. In: Alloza JL, editor. Clinical and Social Pharmacology: Postmarketing Period. Aulendorf, Germany: Editio Cantor; 1985. p. 57-65.

24. Sriwatanakul K, Weis OF, Alloza JL, Kelvie W, Weintraub M, Lasagna L. Analysis of narcotic analgesic usage in the treatment of postoperative pain. JAMA 1983;250:926-9.

25. Weintraub M. Intelligent noncompliance with special emphasis on the elderly. Contemp Pharm Pract 1981;4:8-11.

26. Rucker TD. The formulary process at it relates to drug utilization review. Pharmacy and Therapeutics;1992;17:646-57.

27. Mashford ML. Drug information and therapeutic guidelines: A second opinion before prescribing. In: Alloza JL, editor. Communication in Health Care: Drug Information. III International Medical Pharmaceutical Meeting. Madrid, Spain: Madrid Doctors' Association; 1995. p. 12-6.

28. Tolpin HG. Cost-benefit assessment of pharmaceutical products. Drug Inf $\mathrm{J}$ 1988;22:281-92.

29. Gibbong JH. Evaluation of the Oregon Medicaid Proposal. Washington, DC: U.S. Congress, Office of Technology Assessment; 1992.
30. Yan $Q$, Hunt CA. Preventing adverse drug events (ADEs): The role of computer information sys-tems. Drug Inf J 2000;34:1247-60.

31. Marshall A. Laying the foundations for personalized medicines. Nat Biotechno 1997;15:954-7.

32. Maciá Bobes $C$, Begoña Paredes Ojanguren M, Pilar Poncela Cuesta M, Asunción Alvarez Rueda M, Castaño Fernández G, Venta Obaya R. Serum cortisol concentrations in acute non-critical ill patients, measured in three periods of the day. Med Clin (Barc) 2001;117:254-6.

33. Rylance GW. Prescribing for infants and children. Br Med J (Clin Res Ed) 1988;296:984-6.

34. Leape LL, Woods DD, Hatlie MJ, Kizer KW, Schroeder SA, Lundberg GD. Promoting patient safety by preventing medical error. JAMA 1998;280:1444-7.

35. Conlan MF. Compounding vs manufacturing. Where is the line? Drug Top 1992; October:46-52.

36. Herbal prescriptions. The promises and pitfalls. Consumer Reports; 1999. p. 44-8.

37. Hufford MR. Placebo effects, memory, and the value of real-time data in drug development. App Clin Trials 2002; August:34-43.

38. Alloza JL, Alamo C, Espada M. Analysis of ambiguous terms widely used in the communication process among physicians, pharmacists and patients: A social pharmacology study. Methods Find Exp Clin Pharmacol 2000;22:464.

39. Alloza JL, Lasagna L. A comparison of drug product information in four national compendia. Clin Pharmacol Ther 1983;33:269-77.

40. Moynihan R, Bero L, Ross-Degnan D, Henry D, Lee K, Watkins J, et al. Coverage by the news media of the benefits and risks of medications. $\mathrm{N}$ Engl $\mathrm{J}$ Med 2000;342:1645-50.

41. Szalavitz M. Web sites and misinformation about illicit drugs. N Engl J Med 2001;345:1710.

42. Alloza JL. Social pharmacology of prescribing antidepressants in unregistered indications from the perspective of global medicine. Rev Esp Econ Salud 2010;8:66-72.

43. Alloza JL, Alamo C, Espada M. Analysis of ambiguous terms widely used in the communication process among physicians, pharmacists and patients. Methods Find Exp Clin Pharmacol 2000;22:27.

44. Villanueva P, Peiró S, Librero J, Pereiró I. Accuracy of pharmaceutical advertisements in medical journals. Lancet 2003;361:27-32.

45. Alloza JL. The social pharmacology of "ask your doctor or pharmacist for advice": Concerns about self-medication with OTCs by the consumer and the pharmacist in the pharmacies in the Community of Madrid. Meth Find Exp Clin Pharmacol 2002; 24 (Suppl A): 125

46. Alloza JL. Social pharmacology and drug overregulation. Med Clin (Barc) 2005; $124: 379-82$

47. Schiff GD, Rucker TD. Computerized prescribing: Building the electronic infrastructure for better medication usage. JAMA 1998;279:1024-9.

48. Alloza JL, Espada M, Álamo C, Cuenca E. Informed consent forms, comprehension and volunteering in a controlled clinical trial. Meth Find Exp Clin Pharmacol 1996;18(Suppl.B):117.

Cite this article as: Maiti R, Alloza J. Social Pharmacology: Expanding horizons. Indian J Pharmacol 2014;46:246-50.

Source of Support: Nil. Conflict of Interest: No. 